\title{
Educación Popular Feminista: Clases de Castellano para Mujeres Migrantes Magrebíes
}

\author{
Feminist Popular Education: Spanish Classes for Migrant \\ Women from the Magreb
}

\author{
Alba Sierra Rodríguez * \\ Carlos Peláez-Paz \\ Universidad Complutense de Madrid
}

\begin{abstract}
La feminización de las migraciones y las cadenas globales de cuidados se interrelacionan desarrollando mecanismos vulneradores de derechos que afectan, principalmente, a mujeres migrantes y trabajadoras domésticas. Por ello, parte de esta población sufre desigualdades sociales asociadas a su etnicidad, identidad sexogenérica y clase social. A través de la metodología cualitativa de tipo etnográfica, desarrollamos una investigación con mujeres migrantes magrebíes y trabajadoras domésticas que asisten a clases de castellano en los barrios de San Nicasio y La Fortuna en la ciudad de Leganés (Madrid). En el Estado español, las dificultades en el aprendizaje de una nueva lengua, la falta de contacto con las instituciones públicas y las fricciones con su comunidad de vecinos y vecinas promueven que mujeres con altas capacidades y potencialidades acaben viviendo experiencias de aislamiento, sin llegar a ejercer sus derechos en igualdad de condiciones al resto de la población y por otro lado, la comunidad no se enriquezca de los beneficios de procesos integradores efectivos y buena convivencia. Se analizan situaciones en las que mujeres desarrollan redes de sororidad y ayuda entre compatriotas para suplir las carencias un sistema social de atención a población migrante inefectivo, que no recoge las especificidades derivadas de las necesidades de estas mujeres y, por lo tanto, fracasa al garantizar su acceso a derechos básicos.
\end{abstract}

Descriptores: Educación, Mujer, Educación de adultos, Desigualdad social, Alfabetización.

The feminization of migration and global care chains are interrelated by developing mechanisms that violate rights that affect mainly migrant women and domestic workers. Therefore, part of this population suffers social inequalities associated with their ethnicity, sex-generic identity and social class. Through a qualitative methodology of ethnographic approach, we develop an investigation with migrant women from the Maghreb, who are also domestic workers and attend Spanish classes in the neighborhoods of San Nicasio and La Fortuna in the city of Leganés (Madrid). In the Spanish state, difficulties in learning a new language, lack of contact with public institutions and friction with its community of neighbors promote that women who have high capacities and potentialities end up experiencing isolation, without exercising their rights in equal conditions to the rest of the population and, on the other hand, the community is not enriched by the benefits of effective integrative processes and good coexistence. We analyze situations in which women develop networks of sorority and help among compatriots to fill the gaps of an ineffective attention social system to migrant population, which does not collect the specialties derived from the needs of these women and therefore fails to guarantee their access to basic rights.

Keywords: Education, Women, Adults education, Social inequality, Literacy.

*Contacto: albasierruti@gmail.com

ISSN: 2254-3139

www.rinace.net/riejs/

revistas.uam.es/riejs
Recibido: $\quad 11$ de junio 2017

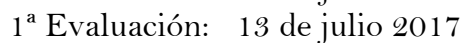

$2^{\text {a }}$ Evaluación: 19 de agosto 2017

Aceptado: $\quad 28$ de septiembre 2017 


\section{Introducción}

Durante bastantes años la cuestión migratoria ha sido uno de los aspectos centrales de las preocupaciones sociales y ha ocupado un puesto central en la agenda política. Sin embargo, parece haber pasado a un segundo plano a consecuencia de la crisis económica, que puso encima de la mesa nuevos fenómenos como los desahucios, el galopante desempleo o la pobreza energética. Este desplazamiento del interés público ha invisibilizado el hecho de que la población inmigrante sufre mayores niveles de desempleo y pobreza que la autóctona. Las mujeres migrantes en particular conforman uno de los sectores de población en el que encontramos mayores situaciones de exclusión social y vulnerabilidad. Aunque en la mayoría de casos les esté reconocida la titularidad formal de derechos, las barreras asociadas a su condición de mujeres, de migrantes y a sus situaciones de vulnerabilidad, impiden que ejerzan en pleno derecho su ciudadanía. La invisibilidad, o al contrario, la estigmatización que sufren determinados perfiles de estas mujeres agravan esta situación.

Concretamente, las mujeres procedentes del Magreb, y más específicamente del Rif, afrontan dificultades asociadas al desconocimiento del idioma, el desconocimiento del funcionamiento de las instituciones españolas y los prejuicios asociados a sus prácticas culturales, entre otros factores. En esta investigación se defiende la hipótesis de que la experiencia de exclusión ligada al género, la etnicidad y la clase social puede ser transformada mediante la acción socioeducativa a través de las potencialidades de las mujeres y su interés por sentirse parte de la comunidad española. En el caso que nos ocupa, estudiamos las potencialidades visibilizadas a raíz de un grupo de mujeres estudiantes de castellano en el barrio San Nicasio en Leganés. Analizamos qué supone para ellas, tanto a escala individual como colectiva, este proceso educativo en el que se priorizan sus intereses y se convierten en motores del cambio y transformación de los contextos vulneradores en los que se desarrolla su vida cotidiana.

\section{Fundamentación teórica}

Partimos de la idea de que las cadenas globales de cuidados, la feminización de las migraciones y la etnificación del trabajo doméstico y de cuidados son algunos de los fenómenos estructurales que subyacen a la experiencia de vida cotidiana de las mujeres migrantes estudiantes de las clases de castellano y nos permiten comprenderla.

Al hablar de cadena global de cuidados nos referimos al fenómeno por el cual se establece una serie de vínculos entre población de todo el mundo basados en una labor de asistencia que puede estar remunerada o no remunerada (Hochschild, 2008). Estas labores de cuidado son desempeñadas principalmente por mujeres, ya que debido a los roles de género asociados a cada identidad sexo-genérica, la identidad mujer sigue identificándose como la responsable del espacio doméstico y las labores ligadas al sistema de reproducción (Harding, 1996).

Autoras como Pérez Orozco (2009) relacionan la cadena global de cuidados con el proceso de feminización de las migraciones en el contexto de la globalización y la transformación de los Estados del Bienestar, ya que la etnificación, es decir, el desempeño de estas labores por parte de un determinado grupo étnico, es un rasgo propio de este proceso. El proceso de feminización es resultado de un entramado social en el que las relaciones se basan en las ideas y valores de una cultura dominante 
heteropatriarcal, con un modelo binario jerarquizado a partir del que se construyen de manera diferenciada los roles y derechos para ciudadanos y ciudadanas (Costa, 2006). Estas ideas, valores y prácticas cristalizan en el desarrollo de intersecciones como la etnia, el género, la clase social que interrelacionadas entre sí dibujan un escenario en el que las mujeres migrantes sufren situaciones de subordinación (Lister, 2003).

En el Estado español, ambos fenómenos sociales son significativos ya que, a raíz de transformaciones sociales, como la incorporación de las mujeres al mercado laboral y la escasa intención de la población masculina en un sistema de conciliación familiar para ocuparse de las labores de cuidado, estas necesidades han sido cubiertas mayoritariamente por mujeres trabajadoras y migrantes solucionando la demanda del país en relación a cubrir estas funciones. Su presencia en el trabajo doméstico ahonda en su situación de subordinación pues, de hecho, la organización social del cuidado resulta en sí misma un vector de reproducción y profundización de la desigualdad social (Rodríguez-Enríquez, 2015). En este sentido, Comas (2015) afirma que las dimensiones de género, clase y etnia se expresan nítidamente en las relaciones laborales de empeladas domésticas, entre otras cosas por la vulnerabilidad de ser migrantes mayoritariamente en situación no regularizada, por la atribución de los cuidados a las mujeres sin implicación masculina, y por la asimetría entre las personas empleadoras y las empleadas.

Nos encontramos ante la necesidad de cuestionar estos valores y prácticas sociales, realizando una lectura crítica que permita la igualdad de derechos y autonomía para toda la población. Para ello, es fundamental introducir, como diría Freire (1969), el diálogo como práctica política en los distintos espacios en los que se construye y refuerza la subordinación y desigualdad y sus discursos legitimadores. Encontramos estos espacios en nuestra cotidianidad, en nuestras familias, trabajos, centros educativos, formales y no formales, lo que facilita generar procesos de cambio en los que se visibilicen distintos saberes y se construya una epistemología común e inclusiva que no responda a diferenciaciones de ningún tipo. Por lo tanto, necesitamos aproximarnos a las experiencias de estas mujeres y recuperar estos saberes, retomando y reconociendo historias poco o casi nunca contadas (Curiel, 2009).

La oportunidad educativa posibilita renegociar las relaciones de inequidad de género, clase y etnia cuando el proceso de enseñanza aprendizaje se dirige a generar activos de las mujeres, entendiendo los activos como los recursos tangibles e intangibles que utilizan las personas para reducir la vulnerabilidad ante situaciones de riesgo y que no sólo se ligan a lo financiero y productivo sino también a los vínculos, el capital social, la identidad y el empoderamiento (Moser, 2011). La vulnerabilidad social, tal y como proponen Kaztman y Filgueira (2006), es también el resultado de la interacción de la estructura de oportunidades y de los activos, así como las prácticas, comportamientos y creencias de los sujetos, que tienen que ver con el uso que hacen de esos recursos.

\section{Metodología}

En el proceso de investigación hemos utilizado una metodología cualitativa de tipo etnográfica, recurriendo a las técnicas de análisis documental, observación participante y entrevista semiestructurada. Tal y como hemos planteado en otros lugares pensamos que la etnografía enriquece los procesos de investigación al promover la reflexión-acción 
de las investigadoras inmersas en ellos (Peláez-Paz, 2013). Gracias a esta metodología hemos podido cambiar nuestra manera de mirar la realidad y nos ha permitido priorizar la voz de las estudiantes de las clases de castellano. Nos ha aportado la posibilidad de centrar parte de la importancia de la investigación en los agentes investigadores como agentes de cambio (Rockwell, 2008).

El análisis documental se ha basado principalmente en el estudio de bibliografía existente en relación a este fenómeno social y en acudir al padrón municipal de Leganés y el análisis de la realidad realizado por la Delegación de Educación del Ayuntamiento de Leganés. La observación participante se desarrolló en las clases de español de un dispositivo municipal en el que participaban mujeres migrantes en los barrios de San Nicasio y La Fortuna, durante los meses de noviembre hasta febrero entre los años 2013 y 2014. El registro de esta observación se ha realizado a través de la elaboración de un diario de campo. Por último, se han realizado entrevistas en profundidad a cinco estudiantes de las clases de castellano del barrio de San Nicasio. La información buscada, se ha basado principalmente en recabar datos sobre tres aspectos fundamentales: la trayectoria migratoria hasta el presente, el trabajo desempeñado en España y su conformidad o quejas sobre su vida diaria.

La selección de la muestra se basó, en primer lugar, en el nivel de castellano. Por funcionalidad, se eligió plantear la posibilidad de realizar entrevistas en un grupo con nivel B1-B2, de manera que entendiesen las preguntas y pudieran explicarse en español, mínimamente. Una vez en la clase, se propuso la entrevista de manera voluntaria y espontánea. En cuanto al perfil laboral, tres de ellas eran empleadas domésticas mientras que las otras dos desempeñaban labores familiares no remuneradas. Las referencias a las entrevistas o la observación participante incluyen nombres ficticios para proteger la privacidad de las estudiantes.

El propósito de la aproximación etnográfica consiste en una reconstrucción o recreación de la realidad social, más que su medición en términos numéricos o estadísticos (Hernández-Sampieri, Fernández-Collado y Barptista-Lucio, 2003). Más que representatividad respecto de un universo de estudio, su interés radica en la producción de información significativa desde el punto de vista de la diversidad de los actores sociales y los contextos en que tienen lugar los fenómenos investigados. El análisis de datos se ha realizado mediante el método de cualificación por categorización poniendo en relación el material empírico con las ideas teóricas que constituye la columna vertebral de la investigación etnográfica (Díaz de Rada, 2011).

\section{Dispositivo y experiencia educativa}

El programa donde se ha realizado la investigación se implementa en Leganés, municipio de la Comunidad de Madrid. El objetivo principal del este programa de educación para personas adultas es dar una respuesta desde las políticas públicas educativas a las necesidades originadas a raíz de la existencia de diversos idiomas en una misma comunidad, nuevas costumbres y tradiciones en contacto directo y la inserción social de población recién llegada. En el municipio residen 189.359 habitantes ${ }^{1}$, de los cuales 24.982 son personas de origen extranjero. El 38 \% de habla hispana y el 62\% con

${ }^{1}$ La fuente es el Padrón Municipal, con fecha a enero de 2013, año en el que se comenzó el trabajo de campo. 
otra lengua materna. La población inmigrante de habla no española está conformada por personas magrebíes. El porcentaje mayoritario lo conforman mujeres analfabetas. En la mayoría de casos, sus ocupaciones son las labores domésticas y las tareas de cuidado, recibiendo un salario por ello al hacerlo en una casa como empleadas o en sus propios hogares. Estas funciones sí que suponen un rasgo común para las estudiantes ya que la gran mayoría están casadas y tiene al menos un hijo o hija. Los rangos de edad de la población con la que se ha desarrollado la observación participante y las entrevistas se encuentran desde los 25 años a los 55 años. En relación a la procedencia de las estudiantes, mayoritariamente son rifeñas (región al Norte de Marruecos), aunque también existe un pequeño porcentaje de mujeres originarias de Tánger, Fez y Casablanca.

Las clases de español para extranjeros y extranjeras se organizan desde la Delegación de Educación del Ayuntamiento de Leganés desde el año 2001 y cuentan con el apoyo de la Universidad Popular de Leganés. El proyecto se coordina con 14 asociaciones de inmigrantes y un equipo multicultural de 40 monitores y educadoras voluntarios conformando un total de 28 grupos que dan clase en diferentes barrios y con diversos horarios por todo Leganés. Existe también la opción online como formación para aquellas personas que tienen problemas con la asistencia. Durante el curso 2013-14 se matricularon un total de 690 personas de las que 510 fueron presenciales y 180 realizaron el curso online.

La acción socioeducativa utiliza las estrategias de la alfabetización y la animación sociocultural como herramientas para la inclusión social, proponiéndose un aumento de la participación social y el asociacionismo entre la población inmigrante. El programa, además de desarrollar esta estrategia educativa en relación a la alfabetización y la inserción social, se encuentra coordinado con servicios de atención al ciudadano, servicios sociales y diversas asociaciones de inmigrantes. Los buenos resultados globales obtenidos en el proyecto favorecen un aumento cuantitativo de la demanda, generalmente debido al boca a boca.

Existen tres fases durante el proceso educativo. La primera, basada en escuchar y conocer en profundidad los intereses y expectativas de las y los estudiantes. La segunda fase, en la que se consolida el espacio-clase como un lugar de encuentro. Por último, la tercera o fase de participación activa en la que se busca el compromiso de las y los estudiantes para realizar más actividades y mejorar la convivencia. Se tiene siempre en cuenta la necesidad de "no juzgar" a las personas que asisten a clase y se otorga durante todo el proceso total importancia al ritmo marcado por ellos y ellas. El profesorado, los educadores y las educadoras entienden que la motivación que quieran poner en juego las mujeres es cuestión suya y el nivel de implicación también.

\section{Perfil de las estudiantes y estructura de género}

La mayoría de mujeres en las clases de castellano son procedentes del Rif, como hemos comentado con anterioridad. Su trayectoria migratoria responde al modelo migratorio clásico en el que es el hombre quien inicia el proceso migratorio. Él sale de su país en primer lugar y una vez estabilizada su situación en el país de acogida, se produce la reagrupación familiar y acude su mujer y el resto de su familia. 
La población rifeña ha estado históricamente marginada y reprimida por el gobierno de Marruecos con quien mantiene un largo conflicto. En el imaginario rifeño se mantiene la guerra de los años veinte en Marruecos, la revuelta del Rif de los años 60 por el olvido y marginación a la que les sometía el gobierno y la represión posterior que dieron paso a los años de plomo. La población, y en especial las mujeres y los niños, acumulan un bagaje de desprotección y pobreza consecuencia de este largo conflicto. Durante años las desapariciones, la tortura y la eliminación física de los disidentes eran frecuentes, posteriormente el olvido de la población y la falta de servicios ha sido la tónica general. Las mujeres y la infancia han sufrido una mayor vulnerabilidad en esta situación, por lo que su casa y su marido eran la primera barrera o herramienta protectora. Como consecuencia su socialización está marcada por una experiencia de repliegue al espacio doméstico y familiar como defensa y amparo.

Este bagaje histórico ha propiciado que la gran mayoría de mujeres que asisten a clase sean analfabetas ya que no han tenido la oportunidad de asistir al colegio en su país de origen. Otro estudio como el de Elvias (2009), que estudia el mismo perfil de mujeres en el municipio de Leganés, apunta a que, en este contexto, la identidad mujer-persona está infravalorada, valorando más las identidades de mujer-madre o mujer-esposa. En estos casos, las familias mantienen un orden de género estricto y jerarquizado en el que las mujeres son las responsables de administrar sus hogares y mantener a sus hijos e hijas y personas mayores a través de las tareas domésticas y los cuidados. De hecho, la asistencia a clases de castellano y los eventos sociales derivados de ésta, en algunos casos, origina un conflicto familiar.

Durante la observación participante, hemos podido observar que muchas de las estudiantes son acompañadas a clase por sus maridos, los cuales observan por la ventana a las mujeres en clase y escuchan los contenidos que se trabajan. Otro ejemplo de control que hemos observado es que. si bien muchas mujeres asisten a las clases de castellano, no todas ellas participan en las excursiones o "días en familia" que se celebran al aire libre. Las mujeres lo explicaban aduciendo que debían pedir permiso a sus maridos para poder hacerlo:

Así no se puede, asi no se puede, tengo una amiga que iba a ir a la excursión del domingo, le dejaron el precio más barato y todo y su marido le dijo que ella con los niños no podía ir, al final fue su marido solo con sus hijos y habia cuatro hombres y resto mujeres en la excursión. (Fátima, 47 años. Nivel bajo de castellano. Trabajadora doméstica. Dos hijas)

\section{Proceso educativo}

\subsection{Motivaciones para la matriculación}

En las entrevistas realizadas, podemos ver que la motivación principal de estas mujeres para optar por las clases de español para extranjeras es evitar la confusión en las actividades cotidianas y desenvolverse correctamente en su entorno: poder hablar con los vecinos y vecinas del barrio, ir solas a hacer la compra o entender al profesor de sus hijos e hijas son algunas de las explicaciones ofrecidas por las estudiantes.

En una de las clases de castellano, una estudiante expresó su deseo de profundizar en el conocimiento del idioma con el objetivo de mejorar su comunicación con la profesora de sus hijos: "Vamos a las tutorías y no entendemos bien a los profesores, tampoco sabemos 
cómo expresarnos, confundimos el futuro con el pasado..." (Aicha, 27 años. Nivel alto de castellano. Casada. Trabajadora doméstica remunerada. Dos hijos).

Las clases de alfabetización la interpretan como una posible clave para el cambio de estas situaciones. El hecho de poder conocer cómo se escribe, cómo se lee y ser parte de las dinámicas de una clase, constituyen uno de las motivaciones centrales de las estudiantes de castellano. Todos estos intereses y motivaciones le otorgan como característica común a los grupos de mujeres el ser altamente proactivos y promover actividades de su interés a las educadoras de las clases de castellano.

Sin embargo, al analizar las experiencias pedagógicas, hemos podido comprobar que existe un autoconcepto erróneo de sus posibilidades y potencialidades ya que muestran una autoestima muy mermada y se ven incapaces de desempeñar funciones o roles nuevos. Las expresiones: "Tengo la cabeza muy dura para aprender", "Es muy difícil para mí" o "No puedo hacerlo" se escuchan frecuentemente durante las clases. En general, existe una representación social común, también en el mundo socioeducativo, que pormenoriza a la mujer musulmana marroquí cuando, realmente, podemos encontrar mujeres con fuertes liderazgos, capacidades y competencias.

Conocer el castellano para poder entablar nuevas relaciones con la población autóctona también ha sido una de las motivaciones visibilizadas por la gran mayoría de ellas.

\begin{abstract}
Antes vivía en Alcorcón y mucho mejor. Aquí (Leganés) siempre te miran raro y nunca te hablan. Antes cuando vivía en Alcorcón todo el mundo, no importa grandes, chicos y viejos, todo el mundo te decía qué tal estás, buenos días, hola. Aquí no. To estoy esperando a que me operen del corazón y me marcho, esto no me gusta. Todo el día en casa, sin hablar con nadie... (Fatoumata, 40 años. Nivel bajo de castellano. Casada. Trabajadora doméstica. Dos hijos)
\end{abstract}

Una parte de ellas, aunque no mayoritariamente, enfoca las clases como un camino hacia una vida mejor. Son conscientes de que hablando castellano podrían optar a mejores trabajos, pero este no es uno de los usos más generalizados de las clases de castellano.

Otra de las motivaciones para ir a clase es lograr un mayor conocimiento sobre su salud para mejorar los tratamientos médicos que siguen. Como veremos posteriormente, en el epígrafe 6.3, la gran mayoría sufren enfermedades que deben tener un seguimiento específico y controlado. Durante una de las clases una de las estudiantes nos comentaba que para ella es imposible acudir a las citas médicas sola. "Mi marido y mis hijos tienen que acompañarme al hospital para hablar con el médico" (Kadija, 45 años. Nivel medio de castellano. Casada. Trabajadora doméstica. Un hijo). De este modo, la clase de castellano aporta a las mujeres un recurso que les permite mejorar su salud y acceder al sistema sanitario, de lo que son conscientes.

\title{
5.2. Espacio educativo de sororidad
}

En el caso de la población magrebí, por razones culturales, las clases han de ser únicamente para mujeres. Este hecho, ha quedado reflejado durante varias ocasiones en la observación participante. Una de las estudiantes de camino al centro donde se realizaba la semana Intercultural de Leganés comentó: "Para los rifeños, estar en un sitio donde hay hombres y mujeres juntos es pecado" (Aouatef. 41 años. Nivel medio de castellano. Casada. Trabajadora doméstica. Tres hijos).

Las clases de castellano para mujeres magrebíes conformado como un espacio femenino al que no acuden hombres estudiantes acaba formando parte de una medida de acción 
positiva dentro de lo que se conoce como un marco dual integrado. Este tipo de diseños tienen el objetivo de promover la igualdad de acceso a derechos entre las estudiantes tal y como afirman Del Pozo Serrano y Peláez-Paz (2010). En consecuencia, las estudiantes perciben el centro de modo positivo, como un espacio de desarrollo personal. Una de las estudiantes afirmaba "Estoy orgullosa de aprender y seguiré estudiando toda la vida" (Ami, 30 años. Nivel alto de castellano. Casada. Trabajadora doméstica remunerada. Dos hijos). Podríamos decir que la posibilidad de compartir tiempo con otras mujeres fuera de sus hogares y conocer a nuevas compañeras constituye una gran influencia para que las estudiantes asistan y se interesen por las clases de castellano.

Este es un componente clave, dado que se genera un espacio en el que las mujeres comparten saberes y experiencias sobre su vida como migrantes magrebíes en Leganés. También comparten recursos materiales, como son los lápices y materiales escolares para la clase y todo tipo de recursos, de lo que hemos conceptualizado como activos. Nos encontramos ante lo que Lagarde (2009) denomina espacio de sororidad, ya que estas mujeres se reconocen como interlocutoras, sin jerarquías entre ellas, desarrollando una relación en la que el principio de reciprocidad está presente y es clave para sus relaciones.

La existencia de espacios de sororidad como este, supone una oportunidad para desarticular falsas creencias y transmitir dudas al grupo clase que no se transmitirían a otras personas o se comunicarían en otros espacios por miedo o vergüenza. Hacerlo en clase no supone ninguna amenaza ya que no es representante ni garante de ninguna institución social, por lo tanto, puede realizarse de manera relajada y sin temores. La respuesta del grupo es en forma de ayuda y apoyo, la puesta en común de alternativas, las dinámicas de resolución de conflictos comentadas en clase, así como la solidaridad garantizada por el grupo "respacializan" a la mujer inmigrante en ámbitos sociales dónde antes no había estado o lo había hecho de manera pasiva. Al igual que Grossberg (2003) entendemos que el afecto entre las personas promueve su agencia, en un proceso de empoderamiento facilitado por la identificación y la pertenencia al grupo-clase. En este proceso, la educadora se perfila como agente facilitador de su formación. La enseñanza de castellano ofrece la oportunidad para trabajar de manera transversal con estas mujeres. Permite, iniciar una relación de confianza a través de la que poder conocer sus fortalezas y potencialidades y abordar las necesidades expuestas por las estudiantes.

En una de las clases, una de las estudiantes rompió a llorar. Sus compañeras comenzaron a preguntarle el porqué. La estudiante respondió que una de sus hijas seguía en Marruecos, intentando entrar a España. Llevaba meses allí, sin que la dejaran pasar y sin que ella pudiera hacer nada para ayudarle. "Ya es mayor de edad, no puedo traerla. Hablamos todos los días y lo único que podemos hacer es llorar” (Mariama, 50 años. Casada. Trabajadora doméstica. Seis hijos. Nivel bajo de castellano).

\subsection{Relaciones domesticas e institucionales}

La experiencia de vida de la población femenina magrebí es un claro ejemplo de cómo existen intercambios comunicativos, desarrollados en determinados ámbitos culturales y sociales, a los que no todas las personas tienen acceso. En su caso, la barrera lingüística impide el ejercicio real de ciertos derechos como puede ser el de salud universal puesto que, aun teniendo el permiso de residencia, la falta de traductores en los hospitales y centros de salud, impide que estas mujeres comuniquen sus malestares al personal médico. 
Durante la investigación realizada, en numerosas ocasiones las estudiantes manifestaban su impotencia al ir al médico y demás servicios del ámbito de la salud. En una de las clases un de ellas comunicaba su indiferencia ante la posibilidad de ir al hospital: "Nunca entiendo lo que me dicen allí, además luego tengo que ir a la farmacia y tampoco comprendo nada" (Aminata, 33 años. Nivel bajo de castellano. Casada. Trabajadora doméstica remunerada. Tres hijos). Esta situación es coherente con la idea planteada por Ugarte (2016) sobre cómo las dificultades en la comunicación, tanto en las relaciones personales como en relación a las instituciones dificultan su integración ya que no pueden mantener relaciones normalizadas en sus barrios con población autóctona $\mathrm{u}$ obstaculizan los trámites administrativos y gestiones con las instituciones del país.

A la barrera idiomática, hay que sumar que la mayoría de estas mujeres responden a lógicas culturales tradicionales, lo que supone una dificultad cuando deben acudir a una consulta médica en la que se encuentren solas ante un hombre, por lo que el derecho a la salud queda obstaculizado de manera doble. Por ello, en la línea de Ugarte (2013) pensamos que incluso en los casos en los que acceden a estos servicios, no se encuentran en igualdad de condiciones con respecto al resto de la población al hacerlo. Durante una de las clases de castellano, una de las estudiantes hacía referencia a un periodo de estancia en el Severo Ochoa. Estuvo ingresada una semana y durante ese tiempo solo su marido y su hijo, que la visitaban en el corto espacio de tiempo de visitas diarias (de media hora a una hora) la traducían al personal médico. El resto del día lo pasaba sola sin hablar con nadie, sin ver la televisión y sin poder comunicar sus dolores o malestares al personal.

En el caso de enfermedades crónicas, como la diabetes, enfermedad muy común entre las estudiantes, tampoco pueden ser independientes en el tratamiento. Las máquinas medidoras de azúcar están en castellano y no comprenden los niveles que indican, por lo que son sus maridos los que se encargan de apuntar los números y conocer su estado de salud. En una de las clases, de nuevo una de las estudiantes afirma que ella no comprende los números de la maquina: "Es mi marido quien lo apunta en la libreta" (Fátima, 45 años. Nivel bajo de castellano. Casada. Trabajadora doméstica. Tres hijos). Ante este caso, nos encontramos en una desprotección total si el marido se ausenta o se desentiende de su tratamiento. De hecho, una de las estudiantes de los niveles básicos murió a causa de una subida de azúcar en Marruecos. Allí se encontraba sola por haber viajado sin compañía. No tenía ningún apoyo que entendiera la medicación y el tratamiento allí. Como podemos ver, este estado de dependencia no solo anula la autonomía de las mujeres y la capacidad de autocuidado, en casos más graves como pudieran ser las enfermedades crónicas, pone en riesgo sus vidas.

En los casos en los que exista violencia machista la probabilidad de detección disminuiría al ser sus propios maridos quienes han de acompañarlas al hospital ya que ellas desconocen los teléfonos de contacto, mayoritariamente carecen de medios económicos para poder ir solas o no pueden comunicar su problemática al personal sanitario. Debido a las dificultades que encuentran estas mujeres para ir solas al médico, este tipo de violencia queda invisibilizada ya que no puede optar por los canales de comunicación institucional ideados para el resto de la población. Por ello, la capacitación sobre vocabulario, recursos y conocimiento del ámbito médico promueve un acercamiento a este tipo de recursos y una menor obstaculización de su uso por parte de estas mujeres. El aprendizaje del castellano desarrolla así, de manera indirecta, la prevención de la violencia machista. 
También es necesario tener en cuenta que muchas de ellas, en sus países de origen, no han tenido la oportunidad de acudir solas al médico o al hospital, por la ausencia de ellos o por el desconocimiento de la lengua árabe. Esto genera que hayan desarrollado un saber popular en relación a la cura de enfermedades y a la gestión de las urgencias médicas, entre otros ámbitos. En una de las clases, una de las estudiantes nos explica cómo reaccionaron su madre y su hermana ante una intoxicación por monóxido de carbono en su casa en Marruecos:

\begin{abstract}
Preparé la bañera con agua caliente. El fuego se apagó y el gas salía y salía y al entrar al baño me desmayé. Me recogió mi hermana y mi madre me dio a oler limones para que despertara. No recuerdo nada de lo que pasó. (Fatoumata, 40 años. Nivel bajo de castellano. Casada. Trabajadora doméstica remunerada. Dos hijos)
\end{abstract}

Una de sus compañeras le pregunta si no fue al hospital y ella contesta que estaban las mujeres solas en casa: "No sabemos árabe ni nada. No podemos ir al hospital solas". El resto de estudiantes, también rifeñas, asienten sin cuestionar la razón: "Cuando llegó, mi hermano me llevó a Urgencias”. Por otro lado, existen situaciones en las que el mal estado de salud o las diferentes enfermedades que las estudiantes puedan sufrir se deben a un desconocimiento de la enfermedad en sí. En ciertos casos, las mujeres se han acostumbrado a ciertos dolores que han normalizado sin interpretar, por desconocimiento, tanto qué es una enfermedad como que existe posibilidad de cura. Este desconocimiento pone en riesgo la salud de la mujer, que puede sufrir enfermedades muy graves sin comentárselo a nadie.

Al hablar de enfermedades y de las diferentes partes del cuerpo que pueden dolerte y por lo que debes ir al médico, una de las estudiantes comenta que le "sale sangre al ir al baño" a defecar sufriendo grandes dolores cada vez que lo hace. Una vez comunicada esta dolencia, el resto del grupo comienza a ofrecer propuestas de manera colectiva, bien basadas en propia experiencia, en el caso de algunas de las estudiantes que ya han sufrido los mismos síntomas, o basadas en la enseñanza de vocabulario y espacios sanitarios a los que puede acudir y ser tratada adecuadamente. Por estas razones, durante el proceso de investigación se consideró tan importante la relación de las estudiantes con los recursos sanitarios y la institución médica. Si bien saben lo que son ciertos recursos como el hospital o el centro de salud, a la vez desconocen aspectos clave, como el nombre de estos o los canales para pedir cita. Ante la pregunta de quién pide cita en casa, las estudiantes contestaron que ellas no, sin especificar quién lo hace. Las respuestas ofrecidas mayoritariamente fueron "Marido, hijas...".

Con respecto a las relaciones sociales mantenidas con el resto de vecinos y vecinas del barrio, las mujeres manifiestan de manera generalizada que apenas mantienen contacto con la comunidad autóctona. En relación a estas percepciones hemos observado que la coexistencia, como forma de sociabilidad, no se traduce en agresividad u hostilidad manifiesta pero sí se tiñe de elementos excluyentes y segregadores al producirse formas de extrañamiento devaluado. Así lo expresa una de las mujeres alumnas del curso, cuando afirma que "las señoras mayores españolas son locas, siempre están igual, "vosotras las marroquíes, vosotras las marroquíes" (Aminata, 34 años. Casada. Nivel medio de castellano. Trabajadora doméstica. Tres hijos). En ese momento, el resto del grupo comienza a verbalizar que el otro día, en el parque, una mujer marroquí discutía con una mujer española por cómo jugaban sus hijos en el parque. La mujer española les dijo que ellas, las marroquíes, no sabían utilizar los columpios bien. Que siempre los 
utilizaban mal. Se repiten las atribuciones homogeneizadoras por razón de origen que construyen una otredad que, en este caso, acaba levantando una frontera en la que parece existir un choque irreconciliable entre modernidad y tradición.

Precisamente durante una de las actividades "fuera del aula", en la que las estudiantes visitan centros médicos u otros servicios públicos del barrio, una de las estudiantes comenta que la gente parece sorprenderse de ellas al verlas en la calle. Su presencia genera lo que parece "incomodidad", "sorpresa" y "rechazo". Estas mujeres están fuera de lugar, no es su sitio, al menos con la visibilidad que les da ir en grupo. Su presencia motiva sorpresa, no es el lugar que deben ocupar, en el que deben estar, o al menos como deben situarse. Su forma de vestir tradicional, el pañuelo y su visibilidad generan en quienes las miran una actitud de distancia, desvalorización y exclusión. Parece que la integración o inclusión en la sociedad española pasa por desprenderse de su atuendo, su simbología e identidad. Activa los mecanismos de auto-confirmación y facilita que se perpetúen las representaciones sociales negativas. Esta situación las vuelve aún más vulnerables y refuerza el ciclo de invisibilidad y aislamiento familiar, donde encuentran protección. Por ello, pensamos que tanto las clases de castellano, como las actividades "fuera del aula" desarrollan procesos de interculturalidad, al visibilizar a las mujeres en espacios en los que se construye como dice Massolo (2003), la organización ciudadana. Es una herramienta a partir de la que pueden revalorizarse y reconocerse socialmente como colectivo y a nivel individual. Por lo tanto, es una oportunidad para generar procesos de participación inclusivos y representativos de toda la población.

\subsection{Prácticas educativas feministas}

La reflexión sobre qué palabras quieren aprender durante las clases de castellano facilita que se pongan en común las necesidades y se propicie un espacio de aprendizaje en el que la dinámica de estudio es colectiva y colaborativa. Otros estudios refuerzan nuestra hipótesis sobre la importancia que tiene desarrollar un proceso de aprendizaje basado en experiencias cotidianas propias a partir de las que son conscientes de la necesidad de aprender la lengua y facilitan la reflexión sobre sus experiencias de vida diarias y el intercambio de las mismas con el grupo (El Achkar et al., 2010).

En una de las clases, una de las estudiantes expresó su frustración al no poder ir a hacer la compra sola y depender de su marido e hijo constantemente: "Quiero aprender a hacer la compra y pagar bien” (Kadija, 45 años. Nivel medio de castellano. Casada. Trabajadora doméstica. Un hijo). Ante este comentario el grupo asintió de manera generalizada, mostrando como necesidad el aprendizaje de los números y el manejo del dinero. Un claro ejemplo de cómo a través de la alfabetización crece la independencia de estas mujeres que podrán actuar sin necesidad de intermediarios, por sí mismas.

Podemos analizar cuando las mujeres empleadas domésticas pusieron en común sus salarios y compararon las tarifas. Ante el bajo salario de una, su compañera una de ellas exclamó: "Te pagan poco, el precio por hora lo debes negociar con tu jefa" (Aminata, 33 años. Nivel bajo de castellano. Casada. Trabajadora doméstica remunerada. Tres hijos). La experiencia de vida, a menudo parecida y compartida, propicia el conocimiento necesario sobre ciertos temas, el intercambio y generación de activos y la empatía adecuada para afrontar cada situación. También la valorización del potencial que poseen tanto individual como colectivamente para solucionar sus dificultades o generar procesos en los que se mejora la situación. La clase facilita en primer lugar, el espacio para contarlo y sentirse escuchadas con el apoyo emocional que merecen. El sentimiento de 
comprensión y acompañamiento a lo largo de estos procesos son factores que autoras como Niño Contreras (2013) reconocen como fundamentales para el desarrollo de un proceso de empoderamiento personal.

Existe la necesidad de introducir aspectos clave en el empoderamiento de las mujeres magrebíes como es el valor que atribuyen a la institución familiar y las potencialidades presentes en ella. En este punto es necesario entender la familia como lo que Collins (2000) llama "locus de resistencia", en el que las mujeres encuentran agencia para crecer $\mathrm{y}$ en el que se sienten apoyadas. Al contrario que los planteamientos propios de los feminismos occidentales que plantean la institución familiar como uno de los ejes de opresión para las mujeres. La reivindicación de la figura de la madre como eje central para el empoderamiento es uno de los ejes discursivos de los feminismos poscoloniales (Jabardo, 2008). La función de cuidadoras es una tarea clave para las estudiantes de castellano en su vida cotidiana. La gran mayoría de ellas tienen al menos un hijo o una hija del que se responsabilizan de manera casi exclusiva. Las tareas de cuidado aún administradas económicamente por sus maridos son elegidas y gestionadas por ellas mismas.

En el caso de las mujeres magrebíes el hecho de encargarse de su familia es una prioridad, como bien expresó una de las estudiantes: "Puedes formarte, pero no a costa de abandonar a tus hijos" (Fátima, 45 años. Nivel bajo de castellano. Casada. Trabajadora doméstica. Tres hijos). En una de las clases, una de las compañeras tuvo que marcharse debido a que su hijo no paraba de llorar y dificultaba al resto de las estudiantes escuchar y entender a la educadora. Al salir, una de las compañeras afirmó: "Las clases están muy bien, pero hace falta un cole para los niños" (Halima, 33 años. Nivel medio de castellano. Casada. Trabajadora doméstica. Dos hijos). Por ello, aquellas estudiantes que no pueden llevar a sus hijos e hijas menores al colegio o la guardería se ven "obligadas" a llevarlos a clase. Esto fortalece ciertos aspectos como puede ser la posibilidad de conocer la interactuación que tienen con los menores, las distintas maneras de educación que ponen en marcha, así como integrar a los pequeños desde edad temprana en el espacio-clase. Sin embargo, la gran mayoría se ha adaptado a aprender en un espacio-clase en el que hay menores presentes que gritan, lloran y ríen y no muestran excesiva preocupación por su presencia. Es más, cuidan a los menores entre todas durante la clase. Esta socialización colectiva de los menores en el aula es un ejemplo de estrategia de las mujeres para poder conciliar su vida como estudiantes y su vida como madres.

\section{Conclusiones}

La etnia, el género y la clase social estructuran una parte de la experiencia cotidiana de las mujeres migrantes marroquíes y dibujan un escenario en el que el riesgo de sufrir vulneraciones de derechos como, por ejemplo, el acceso al derecho sobre su salud, es mayor respecto a hombres, a autóctonas o a personas de clase social con mayor capital económico, cultural y social.

Encontramos barreras explícitas, ligadas en algún caso a situaciones de irregularidad administrativa, y a la estructura de oportunidades laborales y económicas, pero también a obstáculos implícitos o indirectos derivados de las representaciones sociales, la sociabilidad, las actitudes de la población autóctona ante la alteridad, el funcionamiento 
de las instituciones españolas. A estas se añaden otras dificultades como son las propias competencias, el desconocimiento del idioma español o la percepción de no poder desenvolverse en ellas por sí mismas, lo que acentúa esta vulnerabilidad múltiple. Es especialmente significativo el uso que hacen de sus activos y la incidencia de la mayor o menor capacidad de generarlos y valorarlos en su experiencia cotidiana de vida y su trayectoria educativa. En definitiva, la falta de visibilidad de esta población ante ciertas instituciones y la falta de contacto y diálogo social limita el acceso al ejercicio de ciertos derechos. Por su parte, ante esta situación, encontramos que las políticas públicas no atienden de manera totalmente efectiva esta problemática, situándose en muchas ocasiones en planos meramente asistenciales o de control, siendo necesario establecer como finalidad última de la acción socioeducativa promover la participación ciudadana de estas mujeres y el ejercicio de derechos.

Hemos comprobado cómo en las clases de castellano es posible desarrollar una identidad cultural propia en la que tanto los valores como prácticas de las estudiantes se hayan construido a partir del diálogo y el cuestionamiento de la desigualdad. En este proceso de reflexión-acción es importante que la orientación pedagógica ha intentado desarrollar en todo momento la acción socioeducativa desde y para potenciar las competencias y activos de las estudiantes. Por ello, consideramos que la educación popular feminista puede destacarse como herramienta dinamizadora de participación social, promotora de derechos y, finalmente, facilitadora del cambio social, ya que cuenta con posibilidades amplias y reales de transformación.

Existe un fuerte potencial en un modelo de intervención centrado en las motivaciones y competencias de estas mujeres que incorpore una visión alternativa a los modelos centrados en los contenidos meramente lingüísticos. Este modelo trata de promover la expresión de las dudas o los intereses de las estudiantes, así como dinamizar el grupoclase para la visibilización de inquietudes que, en un principio, por falta de confianza o seguridad tanto en ellas mismas como con la maestra o educadora podrían quedar ocultas. En esta línea hemos visto que el equipo del centro ha tratado de desarrollar un enfoque en el que la mujer estudiante es el centro activo y promotor del proceso pedagógico, personalizando tanto el espacio como el contenido trabajado en las clases y garantizando la libertad para expresar todas sus inquietudes. Además, la formación en castellano permite el abordaje de manera transversal de gran cantidad de las necesidades de las mujeres, permitiendo centrar la intervención en las herramientas y potencialidades existentes en ellas mismas como soluciones principales. Una vez se "descubrieron" las grandes potencialidades de las estudiantes, el desarrollo de un proceso de conocimiento basado en las capacidades y las competencias que ellas mismas ofrecían permite la mejora del aprendizaje y el cambio personal y social.

Desde esta perspectiva, un modelo de acción socioeducativa que incorpore un enfoque de derechos, de generación de activos y centrado en las alumnas (y no en los contenidos o las educadoras y maestras), supone el camino hacia un empoderamiento de las mujeres migrantes que garantice su autonomía e independencia, revalorizándolas como personas y visibilizando sus potencialidades para el ejercicio de la ciudadanía en condiciones de igualdad. Para ello, para las estudiantes, la clase debe suponer un espacio propio en el que se promueve un proceso de concientización de su situación y su capacidad para actuar y transformar los contextos de vulneración de derechos en los que se encuentran. Gracias al proceso planteado se desarrolla una devolución de poder a las mujeres 
migrantes y se posibilita, la creación de nuevas identidades y tramas culturales que propiciarán el cambio en las relaciones y la organización social.

Por último, pensamos que este proceso de empoderamiento, personal y colectivo, supone una mejora personal, social y comunitaria, ya que transforma los escenarios cotidianos, incluyendo prácticas sociales basadas en valores e ideas más justas que permiten una convivencia sana y propician los beneficios de una comunidad en equidad de género e intercultural.

\section{Referencias}

Collins, P. H. (2000). Black feminist thought. Nueva York, NY: Routledge

Costa, M. (2006). Distintas consideraciones sobre el binarismo sexo/género. Aparte Rei. Revista de Filosofía, 46, 1-7.

Curiel, R. (2009, junio). Descolonizando el feminismo: una perspectiva desde América Latina y el Caribe. Comunicación presentada en el Primer coloquio latinoamericano sobre praxis y pensamiento feminista. Universidad de Buenos Aires, Argentina.

Comas, D. (2015). Los cuidados de larga duración y el cuarto pilar del sistema de bienestar. Revista de Antropología Social, 24, 375-404.

Del Pozo Serrano, F. J. y Peláez-Paz, C. (2010). La educación para la igualdad: Una necesidad académica y profesional para la acción socioeducativa. En M. Jiménez y F. J del Pozo (Eds.), Propuestas didácticas de educación para la igualdad. (pp. 245-271). Granada: Natívola.

Díaz de Rada, A. (2011). El taller del etnógrafo. Materiales y herramientas de investigación en etnografía. Madrid: UNED.

El Achkar, G., Rodríguez, G., Paz, M. y Rojas, E. (2010). Estrategias de educación popular. Caracas: Ministerio del Poder Popular para Ciencia, Tecnología e Industrias Intermedias.

Elvias, S. (2009). Proyecto de integración y alfabetización de mujeres marroquíes en Leganés. Tabanque, Revista Pedagógica, 22, 31-46.

Freire, P. (1969). Educación como práctica de la libertad. Madrid: Siglo XXI.

Grossberg, L. (2003). Identidad y estudios culturales: ¿no hay nada más que eso? En S. Hall y P. Du Guy (Coords.), Cuestiones de identidad cultural (pp. 148-180). Madrid: Amorrotu Editores.

Harding, S. (1996). Ciencia y feminismo. Madrid: Editorial Morata

Hernández-Sampieri, R., Fernández-Collado, C. y Baptista-Lucio, P. (2003). Metodología de la investigación. Ciudad de México: McGraw-Hill.

Hochschild. A. (2008). La mercantilización de la vida íntima. Apuntes de la casa y el trabajo. Buenos Aires: Katz Editores

Jabardo, M. (2008). Desde el feminismo negro, una mirada al género y la inmigración. En L. Suárez, E. Martín y A. Hernández (Coords.), Feminismos en la antropología: Nuevas propuestas críticas (pp. 39-54). San Sebastián: Ankulegi.

Lagarde, M. (2009). La política feminista de la sororidad. Mujeres en Red, 11, 1-5.

Lister, R. (2003). Citizenship. Feminist perspective. Nueva York, NY: New York University Press. https://doi.org/10.1007/978-0-230-80253-7 
Niño Contreras, L. (2013). Hacia una mayor comprensión del empoderamiento: Las vendedoras ambulantes mixtecas en Tijuana y la participación del estado. Estudios Fronterizos, 14(27), 97-122.

Peláez-Paz, C. (2013). La infancia pobre, marginada y excluida. Aportaciones de la etnografía a la praxis socioeducativa. Educación y Futuro, 29, 81-105.

Pérez Orozco, A. (2009). Miradas globales a la organización social de los cuidados en tiempos de crisis I: ¿Qué está ocurriendo? Santo Domingo: Naciones Unidas.

Rockwell, E. (2008). La relevancia de la etnografía. En E. Rockwell (Ed.), La experiencia etnográfica: Historia y cultura en los procesos educativos (pp. 17-39). Buenos Aires: Paidós.

Rodríguez-Enríquez, C. (2015). Economía feminista y economía del cuidado. Aportes conceptuales para el estudio de la desigualdad. Nueva Sociedad, 256, 30-44.

Ugarte, M. (2013). La atención a la salud reproductiva de las mujeres marroquíes: Análisis cualitativo desde la perspectiva de género (Tesis doctoral). Universidad de Castilla La Mancha, España.

\section{Breve CV de los autores}

\section{Alba Sierra Rodríguez}

Educadora social e investigadora, especialista en estudios de género y derechos de las mujeres. Principalmente ha trabajado en el ámbito del tercer sector en proyectos de prevención de violencia machista entre población joven y con mujeres supervivientes a la violencia de género y el tráfico de personas con fines de explotación sexual. Recientemente, ha colaborado también en la Evaluación de Impacto del Programa por la Erradicación de la mutilación genital femenina en Gambia, como Voluntaria Internacional de las Naciones Unidas y participado en las campañas de promoción de planificación familiar. Becaria de colaboración en la Universidad Complutense de Madrid, actualmente es doctoranda en la Facultad de Educación de la Universidad de Barcelona, investigando los procesos educativos de las mujeres supervivientes a la trata con fines de explotación sexual becada por la Fundación La Caixa. ORCID ID: 0000o003-3016-7963. Email: albasierruti@gmail.com

\section{Carlos Peláez-Paz}

Profesor en el Departamento de Teoría e Historia de la Educación de la Universidad Complutense de Madrid. Educador Social y Antropólogo social y cultural. Miembro del Instituto Universitario IEPALA-Rafael Burgaleta de la UCM, del Instituto de Migraciones, Etnicidad y Desarrollo Social (IMEDES) de la UAM, del grupo de investigación Antropología de las Políticas Social y Culturales (APSYC) y del Grupo de Investigación Estudios en Migración, Identidad, y Ciudadanía (EMIC). Sus líneas de investigación son las de migraciones y convivencia intercultural, derechos humanos, cultura de paz y ciudadanía, exclusión social y animación comunitaria. Ha desarrollado su carrera profesional en el Tercer Sector, trabajando en asociaciones como Semilla, Paz Ahora o la Comisión Española de Ayuda al Refugiado (CEAR) y asesorado a múltiples entidades y administraciones públicas como consultor e investigador dirigiendo al Equipo Ecokimia. Entre otros, destaca la evaluación y formulación del Plan Estratégico de Ciudadanía e Integración (PECI) del Ministerio de Trabajo e Inmigración, en 2011 con IMEDES. ORCID ID: 0000-0002-3905-7595. Email: cpelaez@ucm.es 